\title{
Cubic GaN grown on (0 0 1) GaAs substrate by RF plasma assisted gas source MBE
}

\author{
Li-Wei Sung ${ }^{\mathrm{a}, *}$, Hao-Hsiung Lin ${ }^{\mathrm{a}}$, Chih-Ta Chia ${ }^{\mathrm{b}}$ \\ ${ }^{a}$ Department of Electrical Engineering, Graduate Institute of Electronic Engineering, National Taiwan University, \\ Taipei, Taiwan, ROC \\ ${ }^{\mathrm{b}}$ Department of Physics, National Taiwan Normal University, Taipei, Taiwan, ROC
}

Received 23 July 2001; accepted 21 March 2002

Communicated by R.S. Feigelson

\begin{abstract}
Cubic GaN films have been grown on (001) GaAs substrates by using RF plasma assisted gas source MBE. The cubic GaN films were deposited at different Ga-flux to N-flux ratios that were determined by deposition rates directly. Three growth regimes are defined in the cubic GaN growth diagram. The optical quality of these films was measured by photoluminescence (PL). Micro-Raman scattering was performed to analyze the crystallization of the films. The optimal flux ratio for cubic $\mathrm{GaN}$ grown at $T_{\mathrm{s}}=720^{\circ} \mathrm{C}$ is on the boundary between intermediate Ga stable regime and Ga droplet regime. (C) 2002 Elsevier Science B.V. All rights reserved.
\end{abstract}

PACS: 81.10.- h

Keywords: A1. Crystal structure; A1. Growth models; A3. Molecular beam epitaxy; B1. Nitrides; B2. Semiconducting gallium compounds

\section{Introduction}

As a wide band gap semiconductor, hexagonal GaN has been shown, during the past few years, to be a very useful material for developing both short-wavelength optoelectronic devices and high power electronic devices. Another crystallographic phase of $\mathrm{GaN}$, cubic $\mathrm{GaN}$, is predicted to have several advantages over hexagonal GaN for device applications, including higher carrier mobility,

\footnotetext{
*Corresponding author. Tel.: + 886-2-236-30231, ext. 2429; fax: + 886-2-2363-2442.

E-mail address: sam@epicenter.ee.ntu.edu.tw (L.-W. Sung).
}

easy cleavage, and higher p-type doping efficiency [1-3]. However, epitaxial thin films of this material are difficult to grow and so far their quality has been inferior to hexagonal GaN.

The development of techniques for growing high quality cubic $\mathrm{GaN}$ has been underway for several years. Because cubic $\mathrm{GaN}$ is metastable, its growth conditions are somewhat different from those of hexagonal GaN. The growth of cubic GaN usually utilizes cubic phase $\left(\begin{array}{lll}0 & 0 & 1\end{array}\right)$ substrates to force the deposited GaN into the cubic phase. Cubic GaN has been grown on cubic lattice substrates such as GaAs [4-6], Si [4,7], 3C-SiC [3], and $\mathrm{MgO}$ [8]. For the cubic group III-Nitrides, most effort has been 
focussed on the epitaxial growth [1-4,9] and physical property investigation [10,11] of cubic $\mathrm{GaN}$ films. Research on alloys of cubic nitride semiconductors, specifically AlGaN [12] and InGaN $[13,14]$ are also in progress. Recently cubic GaN LEDs have been successfully realized $[15,16]$.

Attempts to optimize the cubic GaN growth conditions, such as the choice of the V/III ratio, have generally been based on knowledge obtained from hexagonal GaN growth. However, high quality cubic GaN epitaxy can only be done at growth temperatures in the range $700-900^{\circ} \mathrm{C}[1]$, which is much lower than that usually used for hexagonal GaN. In most reports, the transition of RHEED reconstruction patterns from $(2 \times 2)$ to $\mathrm{c}(2 \times 2)[3,4,9,17]$ were used to identify stoichiometric surface conditions [2,9]. However, the actual effect of the V/III ratio on the epitaxial growth of cubic $\mathrm{GaN}$ has not yet been reported. In this study, we report the effect of the $\mathrm{Ga} / \mathrm{N}$ flux ratio on the optical and crystal quality of cubic GaN films.

\section{Experiment procedure}

In this study, cubic GaN was grown on GaAs ( $\left.\begin{array}{lll}0 & 0 & 1\end{array}\right)$ substrates by using an VG Semicon V-80 gas source molecular beam epitaxy (GSMBE) system equipped with an EPI unit-bulb RF plasma cell. Substrate temperature was monitored using an IRCON pyrometer. A typical growth procedure is as follows. Substrate oxide desorption was firstly carried out at $600^{\circ} \mathrm{C}$ under As overpressure with the assistance of atomic hydrogen irradiation. Before the GaN deposition, a 200-nm thick GaAs buffer layer was deposited to flatten the surface. Then, a low temperature cubic GaN buffer layer was deposited on the GaAs surface at $600^{\circ} \mathrm{C}$ at a growth rate of $125 \mathrm{~nm} / \mathrm{h}$. Following the bulk layer growth was proceeded at the substrate temperature ranging from $710^{\circ} \mathrm{C}$ to $750^{\circ} \mathrm{C}$, at a growth rate of $\sim 0.35 \mu \mathrm{m} / \mathrm{h}$. The $\mathrm{V} / \mathrm{III}$ ratio was adjusted by changing the $\mathrm{Ga}$ flux and keeping the $\mathrm{N}$ flux constant, while the N-plasma power was fixed at $300 \mathrm{~W}$ and the corresponding beam-equivalent pressure of the $\mathrm{N}$ species was $1 \times 10^{-4} \mathrm{mbar}$. Photoluminescence (PL) and micro-Raman mea- surements were performed to investigate the epitaxial films. The samples were excited at $12 \mathrm{~K}$ by using a $325 \mathrm{~nm} \mathrm{He-Cd} \mathrm{laser} \mathrm{and} \mathrm{their} \mathrm{PL}$ spectra were recorded with a PMT detector through a SPEX $500 \mathrm{M}$ spectrometer. The excitation source of the micro-Raman was a $514.5 \mathrm{~nm}$ $\mathrm{Ar}^{+}$laser, and the spectra were recorded with a CCD through a DILOR XY spectrometer.

\section{Results and discussion}

To investigate the V/III effect on the cubic GaN deposited by MBE, a series of samples were grown at different V/III ratios. Fig. 1 shows the growth conditions of those samples. Samples marked with open circles represent the films exhibiting Ga droplets on the surface, and the growth conditions are assigned to be in "Ga droplet" regime. In the "intermediate Ga stable" regime, no excessive Ga accumulates on the sample surface. They are marked with close circles. The boundary between "Ga-droplet" and "intermediate Ga stable" regimes is fitted using the $2.83 \mathrm{eV}$ activation energy reported for the excess Ga desorption [18]. On this boundary, the Ga flux was still to be found higher than the $\mathrm{N}$ flux. Therefore, the region below the Ga-droplet regime can be further divided into two regimes, namely, $\mathrm{N}$-stable regime and $\mathrm{Ga}$ stable intermediate regime, as can be seen in Fig. 1. Their

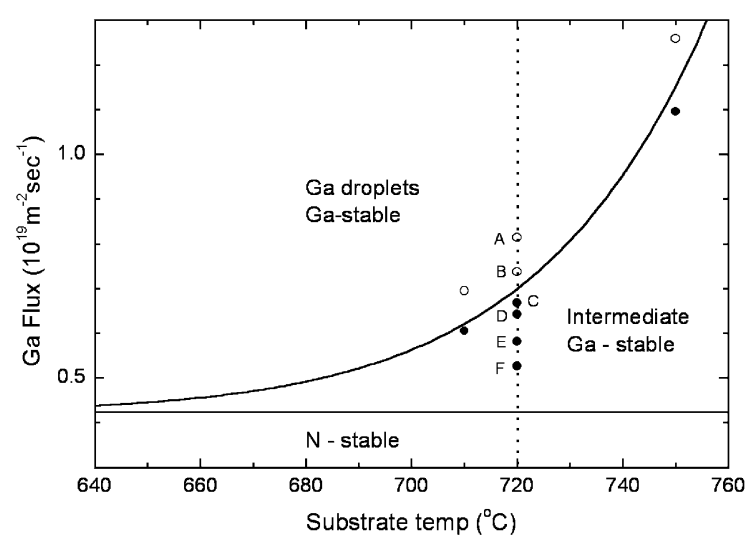

Fig. 1. Surface structure diagram of cubic GaN grown by RF plasma assisted GSMBE. The open circles refer to samples with $\mathrm{Ga}$ droplets. The close circles refer to samples without $\mathrm{Ga}$ droplets. 
boundary was determined by the growth condition that $\mathrm{Ga}$ flux is equal to $\mathrm{N}$ flux. The $\mathrm{V} / \mathrm{III}$ ratio is equal to unity. To measure the flux ratio, cubic GaN film was deposited under the nominal growth procedure but without substrate rotation. In our VG V-80 GSMBE system, the effusion cells are aimed at the edge of the 3 in substrate holder and the deposition uniformity was achieved by rotation. Through the calibration growth, the Ga flux shows $\sim 40 \%$ variation and the $N$ flux shows $\sim 60 \%$ variation through a 3 in substrate. The RF-plasma cell was located opposite to the Ga source on the MBE source flange, as shown in the inset in Fig. 2. The results of sample deposited with identical $\mathrm{V} / \mathrm{III}$ ratio to sample E are displayed in Fig. 2. Curve (a) is the deposited film thickness along scan axis. Curve (b) and (c) are estimated effective $\mathrm{N}$ beam flux and $\mathrm{Ga}$ beam flux, respectively. In the nitrogen stable regime, the $\mathrm{N}$ flux is higher than the Ga flux. When the growth is $\mathrm{Ga}$ deficient, Ga flux limits the $\mathrm{GaN}$ growth rate. When the growth is under $\mathrm{Ga}$ stable regime, available nitrogen limits the growth rate. By fitting the film thickness, the boundary between the $\mathrm{N}$-stable regime and the Ga stable regime was determined. While the Ga droplet regime is distinguished by the appearance of excess $\mathrm{Ga}$ droplets accumulated on surface.

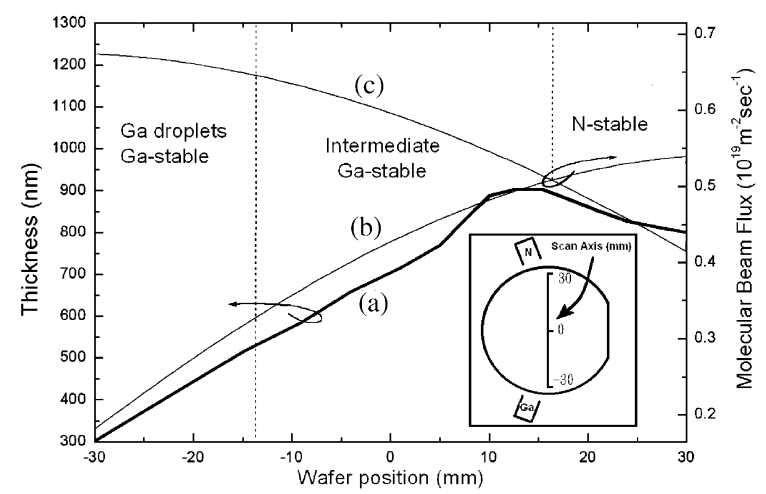

Fig. 2. Curve (a) is the film thickness vs. wafer position result of sample deposited with identical V/III ratio to sample E. Curve (b) is the effective Nitrogen beam flux. Curve (c) is the effective Gallium flux. The inset shows the schematic diagram of RF-plasma cell and Ga cell location on the MBE source flange and scan axis.
To investigate the effect of the $\mathrm{V} / \mathrm{III}$ ratio on the optical characteristics of cubic GaN, low temperature PL measurements were performed. The PL results are shown in Fig. 3. The growth conditions of the samples are indicated in Fig. 1. In the PL spectra, peaks at 357 and $363 \mathrm{~nm}$ are assigned to the hexagonal GaN bound exciton and DAP emission peaks [5,19], respectively. The peak at $379 \mathrm{~nm}$ is assigned to the cubic GaN bound exciton peak. The emissions around 393 and $403 \mathrm{~nm}$ are cubic GaN DAP peaks $[5,19,20]$. The PL spectra of sample A and B grown in the Ga droplet regime show the hexagonal GaN related emission peaks, while the other samples do not. This suggests that hexagonal phase incorporation may result from the Ga droplets. Among the cubic

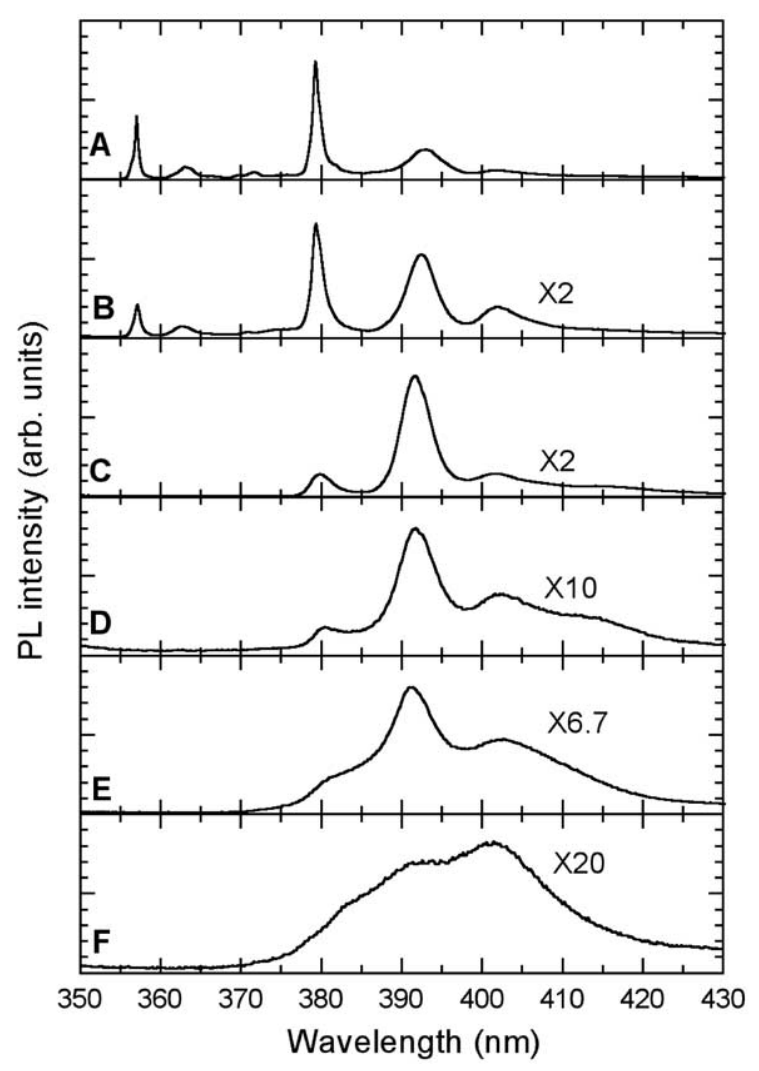

Fig. 3. $12 \mathrm{k}$ PL spectrums of cubic GaN samples A-F. Peaks at 357 and $363 \mathrm{~nm}$ are assigned to the hexagonal GaN bound exiciton and DAP emission peaks, respectively. Peak at $379 \mathrm{~nm}$ is assigned to the cubic $\mathrm{GaN}$ bound exiciton peak. The emissions around 393 and $403 \mathrm{~nm}$ are cubic GaN DAP peaks. 
$\mathrm{GaN}$ related emissions, the bound exciton gets stronger in intensity and narrower in full-width at half-maximum (FWHM) with lower V/III ratio. In addition, the intensity related to the cubic DAP emission peak also decreases with increasing Ga flux. These all reveal that the optical quality of the cubic GaN films can be improved by lowering the $\mathrm{V} / \mathrm{III}$ ratio.

Raman scattering measurements were also used to characterize the crystallinity of the deposited films. Fig. 4 shows the Raman scattering spectra of the six samples. For sample A and B, the Raman measurements were taken from the surface region without $\mathrm{Ga}$ droplets. The peak observed at $738 \mathrm{~cm}^{-1}$ is identified as the longitudinal optical (LO) mode of cubic GaN [21]. The peak at

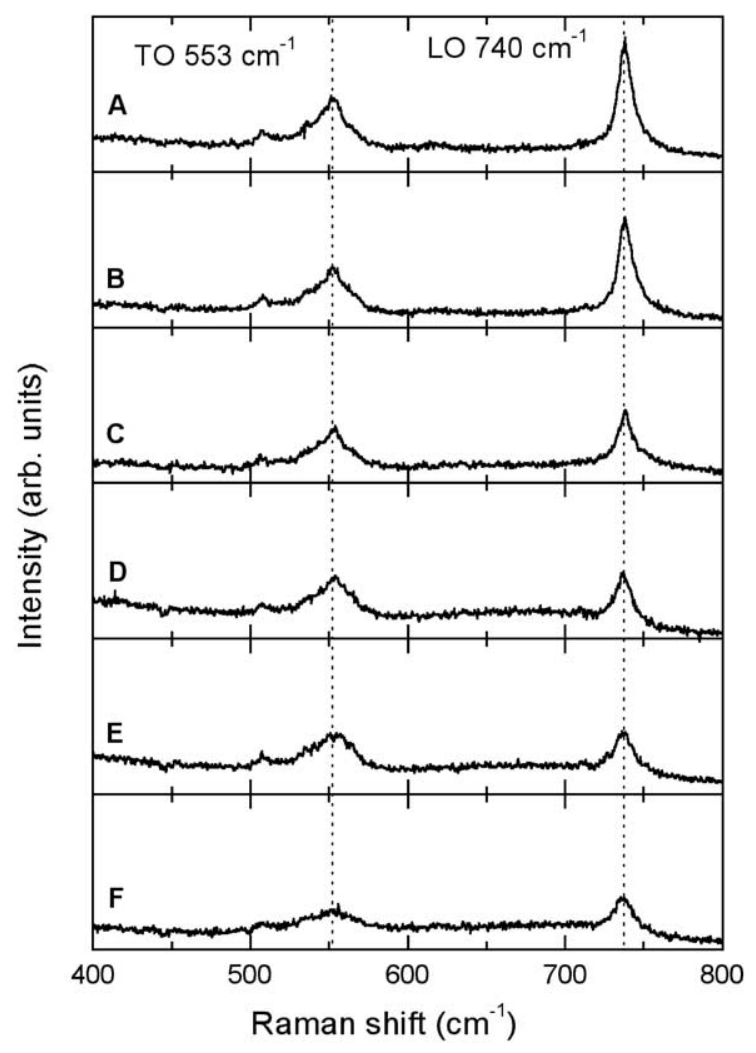

Fig. 4. Room temperature Raman scattering measurement results. The peak observed at $738 \mathrm{~cm}^{-1}$ is identified as the longitudinal optical (LO) mode of cubic GaN. The peak at $553 \mathrm{~cm}^{-1}$ is assigned to the cubic GaN transverse optical (TO) mode.
$553 \mathrm{~cm}^{-1}$ is assigned to the cubic $\mathrm{GaN}$ transverse optical (TO) mode [21]. The peak at $506 \mathrm{~cm}^{-1}$ is the GaAs substrate related scattering. In the spectra, the LO peak shifts toward lower frequency with increasing V/III ratio. It is attributed to the finite crystal effect [22]. In an infinite crystal, only phonons near the center of Brillouin zone contribute to the Raman spectrum. However, when phonon is confined in a space by crystal defects, its momentum becomes uncertain, and phonons with wavevectors deviating from the Brillouin zone center are allowed to contribute to the Raman spectrum. It reveals that crystal defect density increases with increasing $\mathrm{V} / \mathrm{III}$ ratio. The quality of the cubic GaN samples can also be evaluated from the FWHM of the LO peaks [23]. The result shows the same trend. We attribute the quality improvement of the epitaxial films to higher $\mathrm{Ga}$ surface mobility resulting from a decrease in the V/III ratio. When decreasing the $\mathrm{V} / \mathrm{III}$ ratio, the surface coverage of the Ga adlayer will be enhanced. Under this condition, the Ga adatoms are held to the surface by weak, delocalized metallic $\mathrm{Ga}-\mathrm{Ga}$ bonds and thus have higher surface mobility. Therefore, the crystal quality is improved. Similar results had been reported for hexagonal phase $\mathrm{GaN}$ grown on sapphire substrate by using MBE [18].

From the PL spectra of samples A and B, the peaks related to hexagonal phase $\mathrm{GaN}$ clearly indicate its incorporation. However, as can be seen in Fig. 4, no hexagonal phase GaN related $E_{2}$ peak $\left(568 \mathrm{~cm}^{-1}\right)[21,23]$ was observed. This is because the measurement was taken from the surface region without $\mathrm{Ga}$ droplet. To confirm this point, the Raman spectrum taken from the regime under $\mathrm{Ga}$ droplets (removed during measurement) is shown in Fig. 5. A strong and sharp hexagonal $E_{2}$ peak shows that hexagonal phase of $\mathrm{GaN}$ has grown under the Ga droplets [24].

\section{Conclusion}

The effects of the Ga to $\mathrm{N}$ flux ratio on the film quality of cubic GaN grown by RF plasma assisted GSMBE, has been investigated. This study shows that rather than growing under 


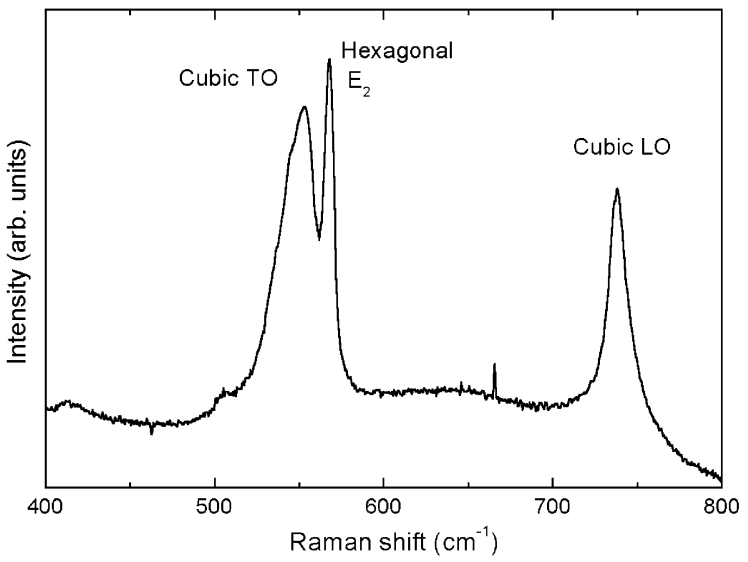

Fig. 5. Raman spectrum taken from the region that was covered with Ga droplet. The droplet was removed before the measurement. A strong hexagonal $E_{2}$ peak $\left(568 \mathrm{~cm}^{-1}\right)$ is observed.

stoichiometric growth condition, growth at the boundary between intermediate Ga stable regime and $\mathrm{Ga}$ droplet regime results in better film quality and without hexagonal phase incorporation.

\section{Acknowledgements}

This work was supported by the National Science Council of the Republic of China under contract No. NSC 89-2215-E-002 -054.

\section{References}

[1] M. Funato, M. Ogawa, T. Ishido, Sz. Fujita, Sg. Fujita, Phys. State. Sol. A 176 (1999) 509.

[2] E. Martinez-Guerrero, F. Chabuel, D. Jalabert, B. Daudin, G. Feuillet, H. Mariette, P. Aboughe-nze, Y. Monteil, Phys. State. Sol. A 176 (1999) 479.

[3] G. Feuillet, H. Hamaguchi, K. Ohta, P. Hacke, H. Okumura, S. Yoshida, Appl. Phys. Lett. 70 (1997) 1025.

[4] K.H. Ploog, O. Brandt, H. Yang, B. Yang, A. Trampert, J. Vac. Sci. Technol. B 16 (1998) 2229.
[5] D.J. As, F. Schmilgus, C. Wang, B. Schottker, D. Schikora, K. Lischka, Appl. Phys. Lett. 70 (1997) 1311.

[6] S. Fujieda, Y. Matsumoto, Jpn. J. Appl. Phys. 30 (1991) L1665.

[7] T. Lei, T.D. Moustakas, R.J. Graham, Y. He, S.J. Berkowitz, J. Appl. Phys. 71 (1992) 4933.

[8] R.C. Powell, N.E. Lee, Y.W. Kim, J.E. Greene, J. Appl. Phys. 73 (1993) 189.

[9] A.P. Limal, T. Frey, U. Kohler, C. Wang, D.J. As, B. Schottker, K. Lischka, D. Schikora, J. Crystal Growth 197 (1999) 31.

[10] S.A. Ding, S.R. Barman, K. Horn, H. Yang, B. Yang, O. Brandt, K. Ploog, Appl. Phys. Lett. 70 (1997) 2407.

[11] M. Giehler, M. Ramsteiner, O. Brandt, H. Yang, K.H. Ploog, Appl. Phys. Lett. 67 (1995) 733.

[12] D.J. As, T. Frey, M. Bartels, K. Lischka, R. Goldhahn, S. Shokhovets, A. Tabata, J.R.L. Fernandez, J.R. Leite, J. Crystal Growth 230 (2001) 421.

[13] O. Brandt, J.R. Mullhauser, A. Trampert, K.H. Ploog, Mater. Sci. Eng. B 59 (1999) 73.

[14] T. Kitamura, S.H. Cho, Y. Ishida, T. Ide, X.Q. Shen, H. Nakanishi, S. Chichibu, H. Okumura, J. Crystal Growth 227-228 (2001) 471.

[15] H. Yang, L.X. Zheng, J.B. Li, X.J. Wang, D.P. Xu, Y.T. Wang, X.W. Hu, P.D. Han, Appl. Phys. Lett. 74 (1999) 2789.

[16] D.J. As, A. Richer, J. Busch, M. Lubbers, J. Mimkes, K. Lischka, Appl. Phys. Lett. 76 (2000) 13.

[17] H. Yang, O. Brandt, M. Wassermeier, J. Behrend, H.P. Schonherr, K.H. Ploog, Appl. Phys. Lett. 68 (1996) 244.

[18] B. Heying, R. Averbeck, L.F. Chen, E. Haus, H. Riechert, J.S. Speck, J. Appl. Phys. 88 (2000) 1855.

[19] A. Georgakilas, K. Amimer, P. Tzanetakis, Z. Hatzopoulos, M. Cengher, B. Pecz, Zs. Czigany, L. Toth, M.V. Baidakova, A.V. Sakharov, V.Yu. Davydov, J. Crystal Growth 227-228 (2001) 410.

[20] Z.X. Liu, A.R. Goni, K. Syassen, H. Siegle, C. Thomsen, B. Schottker, D.J. As, D. Schikora, J. Appl. Phys. 86 (1999) 929.

[21] H. Yaguchi, J. Wu, B. Zhang, Y. Segawa, H. Nagasawa, K. Onabe, Y. Shiraki, J. Crystal Growth 195 (1998) 323.

[22] M. Yoshikawa, Y. Mori, M. Maegawa, G. Katagiri, H. Ishida, A. Ishitani, Appl. Phys. Lett. 62 (1993) 3114.

[23] G. Bentoumi, A. Deneuville, E. Bdaudin, G. Feuillet, E. Martinez, P. Aboughe, Y. Monteil, Thin Solid Films 364 (2000) 107.

[24] M.S. Liu, S. Prawer, L.A. Bursill, D.J. As, R. Brenn, Appl. Phys. Lett. 78 (2001) 2658. 